\title{
Association between early menarche and endometriosis risk: a systematic review and meta-analysis
}

\author{
Mei-Yin $\mathrm{Lu}^{1}$, Jia-Li Niu ${ }^{1}$, and Bin Liu ${ }^{1}$ \\ ${ }^{1}$ Affiliation not available
}

September 7, 2020

\begin{abstract}
Background Early menarche is reported with the risk of endometriosis (EMS) with varying conclusions. Objective To assess the association between menarche age and EMS risk. Search strategy PubMed, Medline and Embase were searched using "endometriosis", "early menarche" , "EMS", "menarche age", and "early menstrual characteristics". Selection criteria Articles that reported the EMS risk in early menarche from Jan 2000 to May 2020 were included. Studies without control group, and lack of data of menarche age were excluded. Data collection and analysis EMS risks in these articles were collected and analysed through in random effects meta-analysis. In addition, subgroup analyses and meta-regressionwere were also performed. Main results A total of 16 studies (8913 EMS cases and 876477 controls) were included in the meta-analysis. The pooled risk of EMS in early menarche (<12 years) was 1.34 (95\% CI: 1.16-1.54), with statistically significant heterogeneity across the studies (I2 $=72.0 \%$ ). Stratified analysis showed that the risks of EMS by earlier menarche was increased in studies started after 2000, and in developing area, which was further confirmed by meta-regression analysis. In addition, higher quality in assessment of the exposure (menarche age) and control of potential confounders can elimenate heterogeneity. Conclusions The earlier age of menarche is a major risk factor of EMS, and its risk has an increasing trend in recent years and in developing countries. Large-scale studies in different ethnic groups are warranted.
\end{abstract}

\section{Hosted file}

3manuscipt-BJOG(1).pdf available at https://authorea.com/users/303430/articles/479618association-between-early-menarche-and-endometriosis-risk-a-systematic-review-and-metaanalysis 


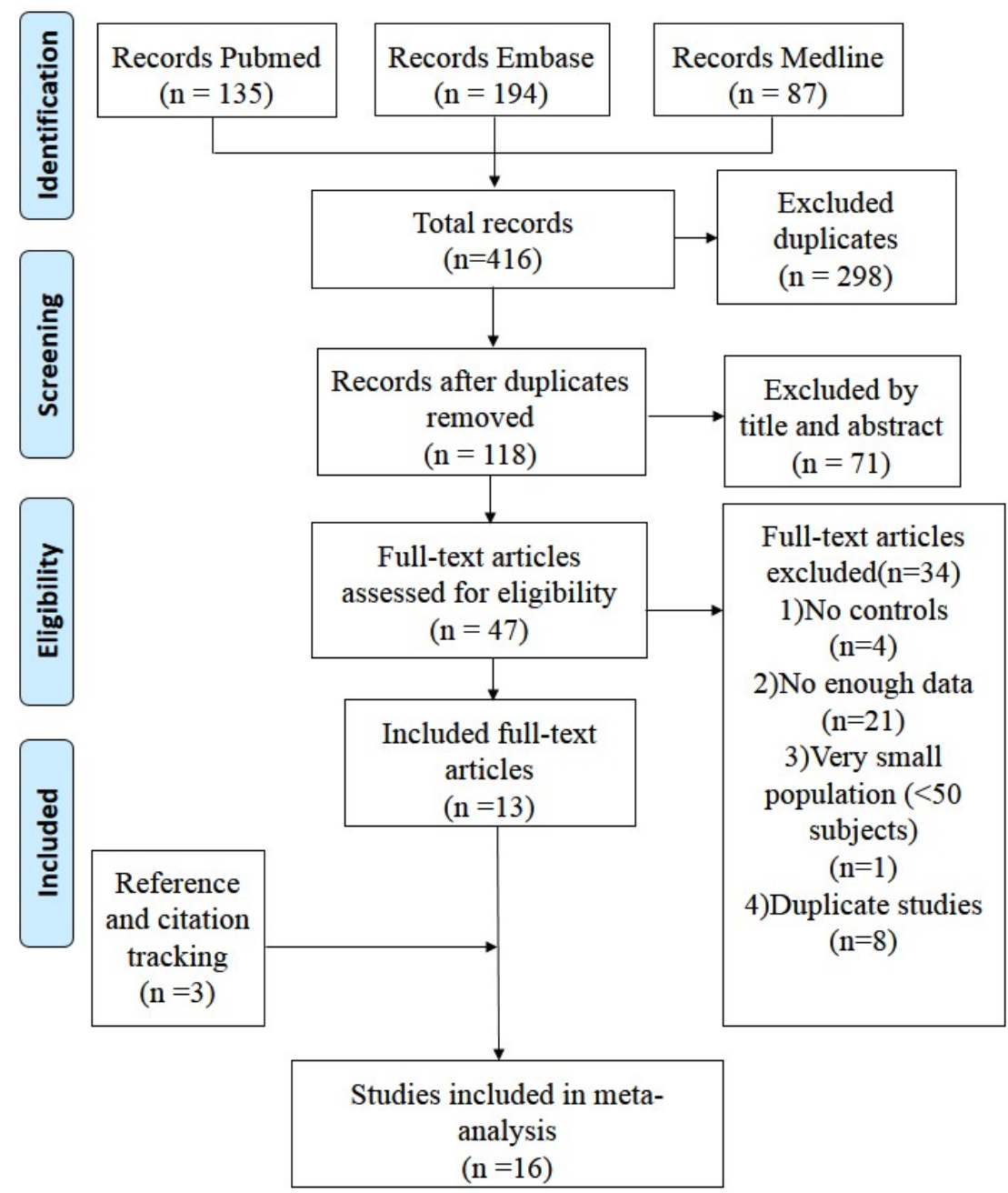




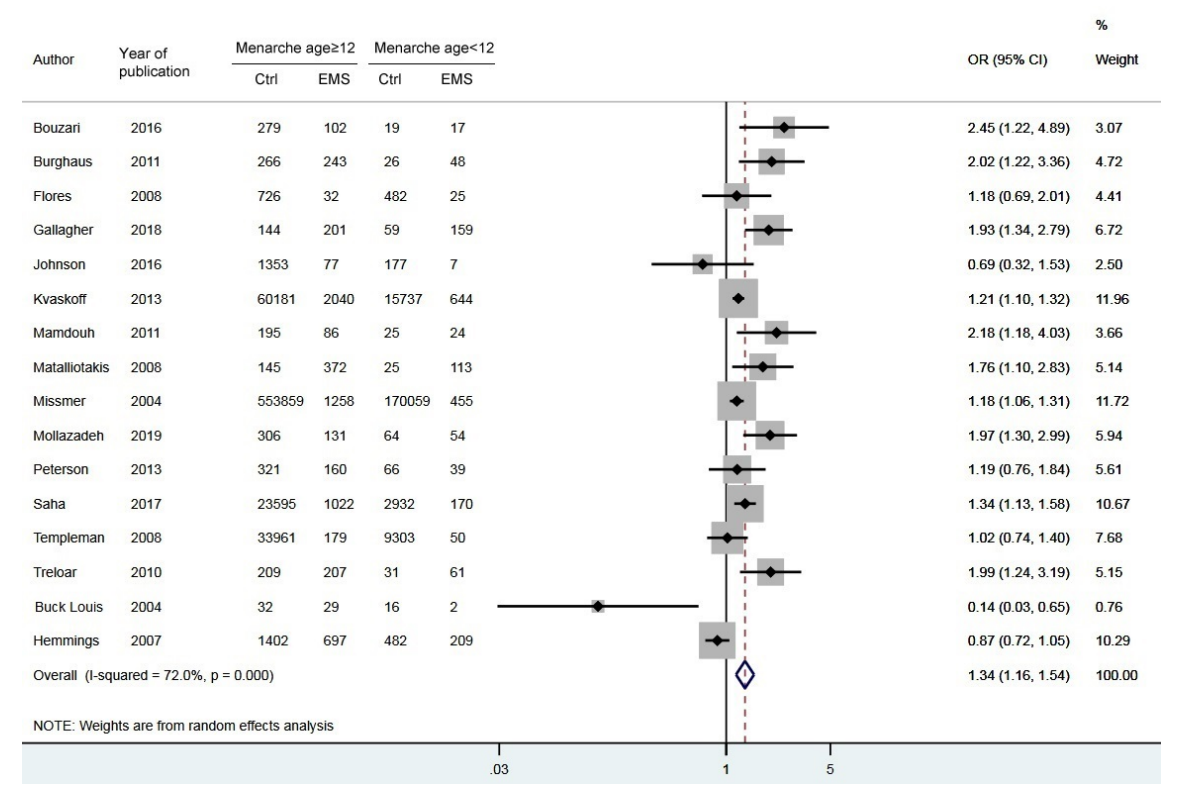

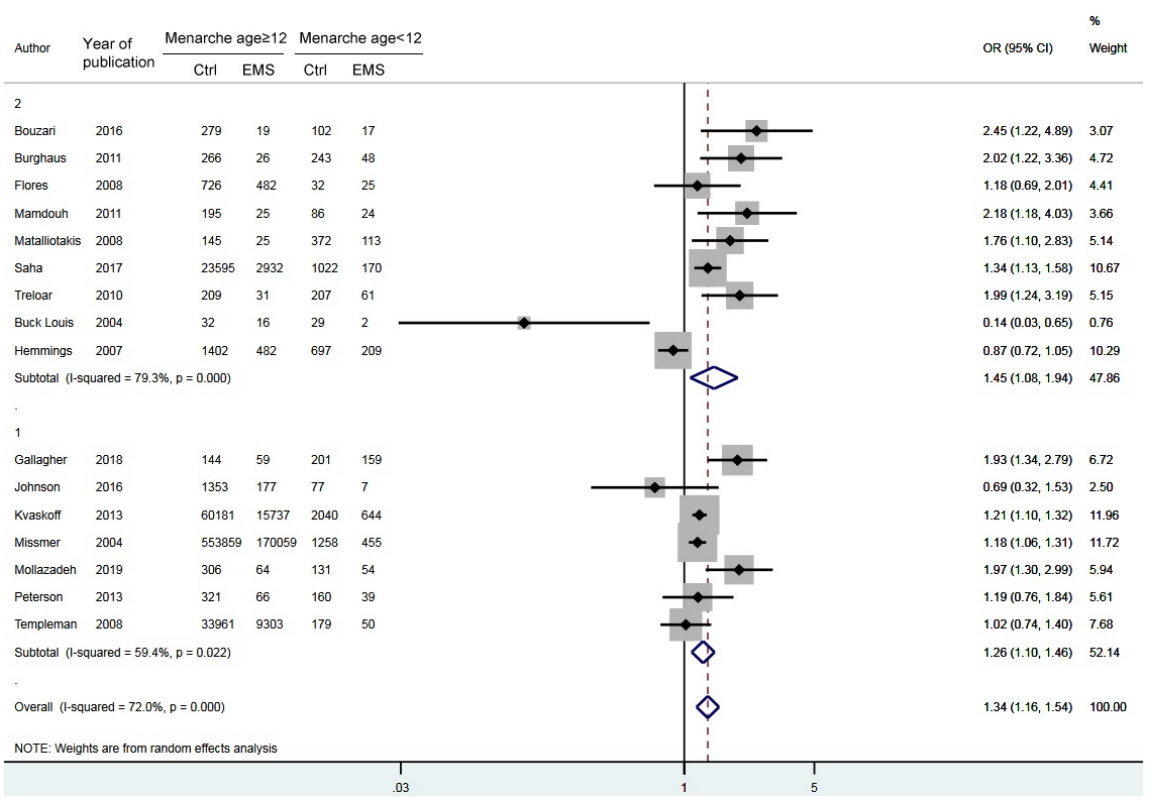

